\title{
Depredación del borrego Ovis canadensis (Artiodactila: Bovidae) y el venado bura Odocoileus hemionus (Artiodactila: Cervidae) por Puma concolor (Carnivora: Felidae) en Coahuila, México
}

\author{
Hugo Sotelo Gallardo ${ }^{1}$, Juan A. García Salas ${ }^{2} \&$ Armando J. Contreras Balderas ${ }^{3}$ \\ 1. CEMEX, Independencia 901-A Oriente, Colonia Cementos. Monterrey, Nuevo León, México. 64520; \\ ugosotelo@hotmail.com \\ 2. Laboratorio de Ornitología, Facultad de Ciencias Biológicas, Universidad Autónoma de Nuevo León. Apartado Postal 425. \\ San Nicolás de los Garza, Nuevo León, México.66450; juan_0305@hotmail.com \\ 3. Consultor Privado. Apartado Postal 425. San Nicolás de los Garza, Nuevo León, México; 66450; ajcb1951@gmail.com
}

Recibido 21-V-2018. Corregido 03-VIII-2018. Aceptado 03-IX-2018.

\begin{abstract}
Predation of bighorn sheep, Ovis canadensis (Artiodactila: Bovidae) and mule deer, Odocoileus hemionus (Artiodactila: Cervidae) by Puma concolor (Carnivora: Felidae) in Coahuila, México. Small and isolated populations of bighorn sheep are vulnerable to predation by mountain lion in habitat sympatric with mule deer. Understanding the specific causes of death and survival is important for the development of conservation strategies for bighorn sheep and other ungulates that share the same habitat, such as mule deer. We evaluated and compared the rate of predation by puma in 12 bighorn sheep (10 females, two males) and 10 adult females of mule deer with radio collars through measure risk program (micromorts). The impact of predation in both populations of herbivores is evaluated through the estimation of densities of sheep and mule deer. $88 \%$ (8/9) deaths by puma in bighorn sheep with an average monthly survival rate of 0.79 and predation rates due to puma range from 0.17 to 0.30 . In mule deer predation due to puma was $83 \%(5 / 6)$ with an average monthly survival rate of 0.86 and predation rates due to puma range from 0.10 to 0.25 , however when comparing the mountain lion depredation rate we found a significant difference between species $(Z=1.826, \mathrm{df}=6, \mathrm{P}=0.05)$. The density in mule deer was $9 \mathrm{x}$ more that bighorn. The bighorn sheep being the prey most selected and the one most affected as the population with the lowest density.
\end{abstract}

Key words: survival rate; bighorn; mule deer; mountain lion predation; Sierra Maderas del Carmen; translocate.

Sotelo Gallardo, H., García Salas, J. A., \& Contreras Balderas, A. J. (2018). Depredación del borrego Ovis canadensis (Artiodactila: Bovidae) y el venado bura Odocoileus hemionus (Artiodactila: Cervidae) por Puma concolor (Carnivora: Felidae) en Coahuila, México. Revista de Biología Tropical, 66(4), 1496-1503.

Entender la causa específica de muerte y supervivencia en las poblaciones de borrego cimarrón (Ovis canadensis; especie sujeta a protección especial en la normatividad mexicana (NOM-059-SEMARNAT-2010; Secretaría de Medio Ambiente y Recursos Naturales [SEMARNAT], 2010), es de utilidad al desarrollar estrategias de manejo para la protección y translocaciones dentro de su hábitat histórico (Schaefer, Torres, \& Bleich, 2000). Reciente evidencia ha sugerido que la depredación tiene un impacto en la dinámica poblacional en plantas y animales (Courchamp, Clutton-Brock, \& Grenfell, 1999). Sin embargo, los escasos estudios de mortalidad por depredación limitan nuestro entendimiento del papel de los depredadores y el impacto que tienen sobre las poblaciones presa.

El borrego cimarrón prefiere hábitats que limiten la capacidad del puma para atacarlos, 
paisajes relativamente libres de vegetación y terreno accidentado que les permiten ver y evitar a los pumas (Shaefer et al., 2000; Logan \& Sweanor, 2001). Sin embargo, el decline de las poblaciones de borrego cimarrón debido a la depredación por puma ocurre donde el ámbito hogareño del borrego cimarrón se traslapa con el del venado bura (Odocoileus hemionus) en densidades adecuadas para proveer presas al puma (Shaefer et al., 2000; Logan \& Sweanor, 2001; Rominger, 2018). Esto debido a que el venado bura es la principal presa del puma en ecosistemas desérticos (Ackerman et al., 1986; Leopold \& Krausman, 1986; Cashman, Peirce, \& Krausman, 1992; Logan \& Sweanor, 2001; Villepique, Pierce, Bleich, \& Bowyer, 2011) y se sospecha que la abundancia de venado bura en rangos de montaña sirve como atrayente para los pumas (Wakeling et al. 2009). Por lo que Rominger (2018) menciona como una de las primeras causas del incremento de la depredación por puma en borrego cimarrón en las últimas cuatro décadas, el incremento de la presencia del felino en hábitats donde históricamente era ausente o raro, en respuesta a la extensiva conversión de ecosistemas abiertos a matorral por la alteración de los ciclos del fuego seguido de la expansión territorial del venado bura. Además, argumenta que el puma que caza en hábitat de borrego cimarrón en baja elevación o pie de montaña difícilmente selecciona venado.

Hallazgos similares se hicieron en un estudio realizado por Schaefer et al. (2000) que evaluaron la causa específica de muerte y supervivencia en una población simpátrica de borrego cimarrón y buras en San Bernardino, California. En donde 26 borrego cimarrón (10 machos y 16 hembras) y 34 venado bura (nueve machos y 25 hembras) fueron dotados de radio collares. Reportaron un $75 \%$ (9/12) de muertes de borrego por puma con una tasa de supervivencia de 0.80 , y para los venados buras encontró un $55 \%$ (5/9) de mortalidad debido a depredación por puma con una tasa de supervivencia de 0.81. Logan \& Sweanor (2001) estudiaron una población de venado bura y población remanente de borrego cimarrón en
San Andrés, Nuevo México, E.U.A. Monitorearon 175 venados con radio collares (91 machos y 84 hembras) durante los años 1987 a 1994 y a 43 borregos con radio collares de telemetría (16 machos y 27 hembras) entre los años de 1985 a 1995, registrando para venado bura $61 \%(62 / 101)$ muertes por puma (35 machos y 27 hembras) y para borrego cimarrón $38 \%(10 / 26)$ bajas a causa de puma (4 machos y 6 hembra). En este estudio concluyen que la depredación por puma fue la mayor causa de muerte para ambas especies.

En 1949, se dio por extirpado al borrego cimarrón de la Sierra Maderas del Carmen, Coahuila, debido a factores como la minería, competencia con borrego doméstico (Ovis aries) y cabras (Capra spp.), fauna exótica como el Berberisco (Ammotragus lervia), enfermedades, cacería no controlada, cercos borregueros, sequía y depredación (Baker 1956; Sánchez 2005). En 2000, CEMEX (Cementos Mexicanos) y la organización no gubernamental Unidos para la Conservación iniciaron un programa de reintroducción del borrego cimarrón en su hábitat histórico en Sierra Maderas del Carmen al norte de Coahuila. El programa empezó con la construcción de un encierro de 5000 ha libre de depredadores, en Pilares, Ocampo, Coahuila. Durante 2001 y 2002 se capturaron a 48 borregos en Sonora, en la reserva del Yaquí, Punta Cirios, San Ramón e Isla Tiburón para liberarlos en el encierro de Pilares. En 2004, se capturó 32 borregos más de la reserva del Yaquí, para liberarlos a estado silvestre en la Sierra Maderas del Carmen (McKinney \& Villalobos, 2005).

Bajo el contexto de esta reintroducción, el objetivo de este estudio es evaluar la tasa de depredación por puma sobre el borrego cimarón y el venado bura, para analizar la selección entre ambas presas del puma en un mismo hábitat, así como el impacto en las poblaciones. Como hipótesis se plantea que el puma es un depredador oportunista que no discrimina entre especies de ungulados simpátricos generando mayor impacto a la especie de menor densidad. El conocer cómo la depredación por pumas afecta las poblaciones del borrego 
cimarrón y venado bura en la Sierra Maderas del Carmen es importante para entender la relación depredador-presa y diseñar estrategias efectivas de reintroducción y conservación de estos ungulados.

\section{MATERIALES Y MÉTODOS}

Área de estudio: La Sierra Maderas del Carmen se ubica al noroeste del estado de Coahuila, México, entre (28 $8^{\circ} 42^{\prime} 18.28^{\prime \prime}$ - 29²1'29.42" N \& 102'22'04.58” $102^{\circ} 55^{\prime} 04.03$ ” W). La parte norte y noroeste del área está limitada por el río Bravo, donde colinda con el Parque Nacional Big Bend; al oeste y al sur limita con por la carretera Melchor Múzquiz - Boquillas del Carmen y al este con el camino de terracería El Melón- La Linda. Políticamente forma parte de los municipios de Ocampo, Acuña y Múzquiz. El rango de elevación va desde los $500 \mathrm{~m}$ en los márgenes del río Bravo hasta los 2720 msnm en los picos más altos de la sierra. La precipitación se presenta principalmente en verano que varía de 100-200 mm en las colinas bajas hasta 200-300 $\mathrm{mm}$ en las partes más altas. La temperatura promedio anual es de $22{ }^{\circ} \mathrm{C}$, con temperaturas en invierno por debajo de $\operatorname{los} 0{ }^{\circ} \mathrm{C}$. Las comunidades vegetales presentes son matorral desértico chihuahuense (micrófilo, rosetófilo, halófilo y gypsófilo), matorral submontano, zacatal y bosque de montaña (pino, encino y oyamel) que se presentan de acuerdo al gradiente altitudinal (Secretaría del Medio Ambiente, Recursos Naturales [SEMARNAT], 2013).

Los borregos y venados fueron capturados con la técnica de rifle de red desde un helicóptero (Wehausen, 1996; Kamler, Lee, de Vos, Balard, \& Whitlaw, 2002; Rominger, Whitlaw, Weybright, Dunn, \& Ballard, 2004; McKinney, Smith, \& de Vos, 2006a), del criadero de vida silvestre de Los Pilares, Ocampo, Coahuila en noviembre del 2014 (permiso DGVS/09/04067). A todo los individuos se les cubrieron los ojos durante el manejo para reducir el estrés de captura, una vez contenidos, médicos veterinarios tomaron muestras de sangre, se les inoculó antibióticos, antiparásitos, des-inflamatorios, vitaminas, un reforzamiento del sistema inmunológico y aretes de identificación. Finalmente, individuos mayores de tres años de edad se equiparon con radio collares de telemetría modelo MOD-600 con frecuencia VHF de seis dígitos y sensor de mortalidad de cuatro horas de activación (Telonics Inc., Mesa AZ., E.U.A.). Previo a la liberación en estado silvestre y con el fin de reducir el estrés de captura y la dispersión en el grupo de borrego cimarrón, se construyó, al pie de la Sierra Maderas del Carmen, un corral de adaptación de 1 ha con un cerco de $2.4 \mathrm{~m}$ de alto con doble línea eléctrica para evitar el ingreso de depredadores. El grupo de borregos se confinó por seis días de climatización, suplementados con agua y alfalfa. Los venados buras se mantuvieron en cautiverio cinco días en otro corral de climatización de 4 ha con un cerco de $2.4 \mathrm{~m}$ de alto ubicado a $10 \mathrm{~km}$ del corral de los borregos en el valle oeste de la Sierra Maderas del Carmen, también se les brindó agua y alfalfa, y se liberaron sin presión humana. El monitoreo de los individuos reubicados se realizó con ayuda de un receptor TR-4 (Telonics Inc., Mesa AZ., E.U.A.) durante los primeros seis meses después de su liberación con una intensidad de tres a cinco veces por semana.

Una vez en vida silvestre, al recibir señal de mortalidad se localizó al individuo lo antes posible para investigar las causas de muerte. La mortalidad a causa de puma se determinó por las características únicas de esta especie en su ataque que incluye: arrastrar y cubrir a su presa con material vegetal, arena, piedras o el mismo pelo de la presa, así como marcas de colmillo profundas en cuello o nuca, extracción del rumen, huellas de patas, excretas, marcas en suelo, y cuando termina de alimentarse de los órganos y carne, continua con las costillas, huesos de la cabeza y fémur (Krausman \& Ables, 1981; McKinney, 1996; Rominger et al., 2004).

Se calculó la tasa de supervivencia y depredación por puma por medio de micromort (Heisey \& Fuller, 1985; Haynes, Rubin, Jorgensen, Botta, \& Boyce 2000; Logan \& Sweanor, 2001; Kamler et al., 2002; Rominger et al., 2004). Este método se utiliza para estimaciones 
de tasas de supervivencia y causas específicas de muerte en animales con radio trasmisores, donde las tasas son estimadas por el número de días trasmitidos, el número de mortalidades debido a una causa en particular, y el número de días en intervalo de tiempo. La causa específica de muerte por puma se obtuvo de dividir el número de mortalidades por puma entre el total de mortalidades durante el estudio (Heisey \& Fuller, 1985).

$$
\hat{S}_{i}=\frac{X_{i}-Y_{i}}{X_{i}}
$$

Dónde: $\hat{\mathrm{S}}_{i}$ es La tasa de supervivencia, $\mathrm{X}_{\mathrm{i}}$ total de número de días transmitidos $\mathrm{y}_{i} \mathrm{Y}_{i}$ número total de muertes en intervalos de tiempo $i$.

Una vez obtenido el cálculo de las tasas mensuales de depredación por puma en los ungulados y con base en la prueba de normalidad Kolmogorov-Smirnov se utilizó una prueba no paramétrica de Wilcoxon $95 \%$ I.C. en el programa estadístico PAST, donde Ho = no hay diferencia en selección de presa y $\mathrm{Ha}=$ sí hay diferencia en selección de presa.

Previo a la translocación y con el fin de demostrar la hipótesis, se estimaron las densidades de los ungulados antes de la liberación en Sierra Maderas del Carmen, en el mes de octubre 2014. Con base en el comportamiento, ciclo biológico de cada especie y el acceso al terreno, se empleó diferente metodología para cada especie. El censo de borrego cimarrón se realizó a través de conteo físico (Simmons \& Hansen, 1980; Ross, Jalkotzy, Festa-Bianchet, 1997). Se fijaron ocho estaciones de observación en áreas representativas para el borrego cimarrón, los cuales se recorrieron con una cuatrimoto por la mañana $(07: 00 \mathrm{~h}-10: 00 \mathrm{~h}) \mathrm{y}$ por la tarde (16:00 h-19:00 h), localizando a los individuos por medio de telemetría y observación directa con ayuda de unos binoculares Zeiss $($ 8 842 y monocular Sawarovski@ modelo ATX 20-60x85, se contó el total de borregos observados y se dividió entre el máximo ámbito hogareño $59.05 \mathrm{~km}^{2}$ (Velázquez, 2012). En el caso del venado bura, se realizaron conteos nocturnos con ayuda de luz artificial en tres transectos fijos de $400 \mathrm{~m}$ aproximadamente de ancho, que en total suman $16.8 \mathrm{~km}^{2}$ aproximadamente (Villarreal, 1999; Lancia, Kendall, Pollock, \& Nichols, 2005).

\section{RESULTADOS}

A 12 borregos (10 hembras y 2 machos) y a 10 venados bura hembras se les incorporaron collares de telemetría. De noviembre 2014 hasta abril 2015 se registraron 1241 días transmitidos en los 12 borregos y 1328 días transmitidos en las 10 venadas bura. La causa específica de muerte por puma en borrego fue $88 \%(8 / 9)$, la tasa promedio diaria de supervivencia en borrego cimarrón fue de $0.9914 \pm$ $0.0016 \mathrm{SD}$, la tasa promedio mensual de supervivencia fue de $0.79 \mathrm{y}$ en la tasa mensual por depredación por puma tuvo rangos de 0.17 a 0.30 (Cuadro 1). En venado bura, la causa específica de muerte por puma fue $83 \%(5 / 6)$. La tasa media de supervivencia diaria en venado bura fue de $0.9944 \pm 0.0043 \mathrm{SD}$, la tasa media mensual de supervivencia fue de $0.86 \mathrm{y}$ la tasa de depredación mensual por puma tuvo rangos de 0.10 a 0.17 (Cuadro 1). En las densidades, se estimó 0.77 borrego $/ \mathrm{km}^{2}$ y 7.0 venado $/ \mathrm{km}^{2}$. En el análisis de comparación entre las tasas de depredación por puma usando la prueba de Wilcoxon 95 \% I.C., encontramos una diferencia significativa $(Z=1.826 ; \mathrm{df}=6 ; \mathrm{P}=0.05)$ siendo el borrego cimarrón la presa más selecta y de menor densidad.

\section{DISCUSIÓN}

Se encontró que las tasas de supervivencia y causa de muerte por puma tienen valores parecidos entre el borrego cimarrón y venado bura, por lo que se demostró que el puma no discrimina entre especies de ungulados que comparten el mismo hábitat. Logan \& Sweanor (2001) mencionan que el puma no premedita la selección de presa, sino que, como depredador oportunista, toma ventaja de la vulnerabilidad y densidad de la presa. Sin embargo, al comparar la depredación mensual entre estas especies se observó una diferencia significativa, siendo el 
CUADRO 1

Tasas de supervivencia y mortalidad en Ovis canadensis y Odocoileus hemionus con radio collares en Sierra Maderas del Carmen

TABLE 1

Survival and mortality rates of Ovis canadensis and Odocoileus hemionus with radio collar in Sierra Maderas del Carmen

\begin{tabular}{|c|c|c|c|c|c|c|c|c|c|c|c|c|c|c|}
\hline \multirow{3}{*}{$\begin{array}{l}\text { Mes/año } \\
\text { Nov/2014 }\end{array}$} & \multirow{2}{*}{\multicolumn{2}{|c|}{$\begin{array}{c}\text { Días } \\
\text { transmitidos }\end{array}$}} & \multicolumn{6}{|c|}{$\begin{array}{c}\text { Tasas diarias en Ovis canadensis } \\
\text { y Odocoileus hemionus }\end{array}$} & \multicolumn{6}{|c|}{$\begin{array}{c}\text { Tasas mensuales en Ovis canadensis } \\
\text { y Odocoileus hemionus }\end{array}$} \\
\hline & & & \multicolumn{2}{|c|}{ Supervivencia } & \multicolumn{2}{|c|}{$\begin{array}{l}\text { Depredación } \\
\text { por puma }\end{array}$} & \multicolumn{2}{|c|}{$\begin{array}{l}\text { Otra causa } \\
\text { de muerte }\end{array}$} & \multicolumn{2}{|c|}{ Supervivencia } & \multicolumn{2}{|c|}{$\begin{array}{l}\text { Depredación } \\
\text { por puma }\end{array}$} & \multicolumn{2}{|c|}{$\begin{array}{l}\text { Otra causa } \\
\text { de muerte }\end{array}$} \\
\hline & 360 & 300 & 0.994 & 0.996 & 0.005 & 0.0034 & 0 & 0 & 0.83 & 0.90 & 0.17 & 0.10 & 0 & 0 \\
\hline $\mathrm{Dic} / 2014$ & 310 & 279 & 0.990 & 0.996 & 0.009 & 0.0034 & 0 & 0 & 0.70 & 0.88 & 0.30 & 0.12 & 0 & 0 \\
\hline Ene/2015 & 217 & 248 & 0.990 & 0.991 & 0.009 & 0.0080 & 0 & 0 & 0.71 & 0.75 & 0.29 & 0.25 & 0 & 0 \\
\hline $\mathrm{Feb} / 2015$ & 140 & 196 & 0.992 & 0.988 & 0.007 & 0.006 & 0 & 0.006 & 0.80 & 0.66 & 0.20 & 0.17 & 0 & 0.17 \\
\hline Mar/2015 & 124 & 155 & 0.991 & 1 & 0 & 0 & 0.008 & 0 & 0.75 & 1 & 0 & 0 & 0.25 & 0 \\
\hline Abr/2015 & 90 & 150 & 1 & 1 & 0 & 0 & 0 & 0 & 1 & 1 & 0 & 0 & 0 & 0 \\
\hline
\end{tabular}

borrego cimarrón la especie más selecta y de menor densidad, impactando en el establecimiento del grupo. La estrategia anti-depredador del borrego cimarrón es más efectiva al acoso por lobos que al asecho por pumas, por lo que la expansión de los pumas debido a la extirpación del lobo ha resultado en una trampa ecológica para los borregos donde los cambios abruptos inducidos por el hombre sobrepasan el tiempo de adaptación evolutiva de la especie (Rominger, 2018). La depredación por puma en especies de baja densidad también ha sido reportada en la Patagonia Chilena por Elbroch \& Wittmer (2013), donde identificaron pumas que seleccionan especies raras y de menor abundancia en un sistema multi-presa impactando la recuperación de la población de las presas menos abundantes. Resultados similares fueron reportados por Schaefer et al. (2000), donde demostraron que la depredación por puma ha disminuido poblaciones nativas de borrego cimarrón (taxón en vías de extinción; Villepique et al., 2011; Romiger, 2018) en San Bernardino, California y también por Logan \& Sweanor (2001), quienes encontraron que el mayor factor limitante en la población de borrego cimarrón de San Andres, Nuevo México, fue la depredación por puma, al grado que en 1997 fue declarado biológicamente extirpada por causas de depredación por puma, en ambos lugares los borregos cimarrones y los venados buras son simpátricos.

Algunos autores como Leopold \& Krausman (1986), Kamler et al. (2002), Rosas-Rosas, Valdez, Bender, \& Daniel (2003), Holl, Bleich, \& Torres (2004) y McKinney et al. (2006a) sugieren que el puma cambia a presa alternativa (borrego cimarrón) cuando su presa principal (venado bura) disminuye en abundancia. Sin embargo, en el caso de este estudio, la población de venado bura es $9 x$ mayor que la del borrego cimarrón y se demostró que las causas específicas de muerte por puma son cercanamente similares, por lo que el puma no necesita una disminución de su presa principal para cambiar a presa alternativa.

En el primer día de la liberación se observó al grupo de borregos liberado unirse con algunos individuos del grupo fundador en su rango de invierno que es de baja elevación o pie de montaña (Wehausen, 1996) hábitat que comparten con los venados bura en Sierra Maderas del Carmen. El puma como depredador oportunista, toma ventaja de la densidad y vulnerabilidad de la presa (Logan \& Sweanor, 2001) y se asume que, como resultado inmediato de la translocación, se aumentó el número de presas en el área que resultaron más vulnerables por la desorientación en su nuevo hábitat, lo que favoreció a los pumas, ya que aparte de las ocho 
mortalidades por puma del grupo del 2014 se encontraron tres hembras depredadas del grupo fundador. Resultados semejantes fueron encontrados en Sierra Catalina, Arizona donde una primer translocación de borrego cimarrón en el 2013 se reportaron 30 mortalidades de las cuales 15 fueron a causa de depredación por puma, en los primeros cuatro meses de la liberación (Rominger 2018). La liberación de adaptación cumplió el objetivo de mantener al grupo unido ya que el tamaño del grupo se asocia con un menor riesgo de depredación (Mooring, 2004) debido a que los borregos cimarrones viven y se alimentan en conjunto como comportamiento anti-depredador (FestaBianchet, 1991; Ross et al., 1997; Logan \& Sweanor, 2001). Sin embargo, no redujo la depredación por puma; Dickens, Delehanty, \& Romero (2010) mencionan que después de una captura, manejo y transportación el siguiente proceso es frecuentemente el cautiverio previo a la liberación y esta última fase en algunos animales silvestres puede causar estrés crónico afectando significativamente la habilidad de escapar del depredador en los siguientes días de la liberación. En diferentes translocaciones de borregos cimarrón los pumas representan uno de los principales factores de decesos al poco tiempo después de la liberación (Chow, 1991; Rominger et al., 2004; McKinney et al., 2006b; Janke, 2015.

En uno de los sitios de depredación de borregos por puma, se encontraron los restos de una hembra con radio collar junto a un macho de la misma liberación identificado por el arete y a $1 \mathrm{~km}$ al norte aproximadamente fueron localizadas dos hembras más, depredadas por puma en un mismo cañón a pocos metros una de la otra. Por la intensidad, el área y el tiempo de depredación se asume que se trata de un puma especialista. Registros de pumas especialistas han sido reportados por Hoban (1990), Ross et al. (1997), Logan \& Sweanor (2001), Ernest, Rubin, \& Boyce (2002).

La abundancia de venado bura se debe considerar como un factor potencial en la influencia de depredación por puma sobre los borregos cimarrones (McKinney et al., 2006b) a que las poblaciones de puma no persisten en lugares donde las densidades de venado son menores a 1 venado $/ \mathrm{km}^{2}$ (Cashman et al., 1992), se sugiere en el futuro, realizar translocaciones de borrego cimarrón en áreas de bajas densidades de venado bura y por ende baja presencia de puma, ya que el decline de las poblaciones de borrego cimarrón, debido a depredación por puma ha sido reportada en casi todos los estados y provincias donde estas especies coexisten (Rominger, 2017). Esto podría dar una posible solución a la hora de seleccionar el hábitat potencial para futuras translocaciones de borrego cimarrón, debido a que, la depredación por puma puede generar el efecto Allee (dependencia inversa de densidad; Mooring, 2004; Bourbeau-Lemieux, Festa-Bianchet, Gillard, \& Pelletier, 2011). Esto afecta la dinámica de la población, haciendo al grupo susceptible al colapso por la temprana depresión en las tasas de crecimiento, reduciendo la posibilidad del establecimiento de la población de borregos, incluyendo hábitat histórico, calidad de hábitat y terreno de escape, ya que se sospecha que la abundancia de venado bura en rangos de montaña sirve como atrayente para los pumas (Wakeling et al., 2009) y puede permanecer en áreas donde el borrego cimarrón es simpátrico con el venado bura en densidades abundantes (Schaefer et al., 2000; Wakeling et al., 2009).

\section{AGRADECIMIENTOS}

Agradecemos a CEMEX por el financiamiento del estudio y por esta iniciativa de conservación y al personal de La Reserva Natural El Carmen y a los revisores por su valiosa aportación. Este trabajo es parte del requerimiento para obtener el grado de Doctor en Ciencias en Manejado de Vida Silvestre y Desarrollo Sustentable en la Facultad de Ciencias Biológicas de la Universidad Autónoma Nuevo León (H.S.G.).

\section{RESUMEN}

Pequeñas y aisladas poblaciones de borrego cimarrón (Ovis canadensis; especie sujeta a protección especial) son 
susceptibles a la depredación en hábitat simpátrico con venado bura. Entender las causas específicas de muerte y supervivencia es importante para el desarrollo de estrategias de conservación para el borrego cimarrón y otros ungulados que comparten el mismo hábitat, tal como el venado bura (Odocoileus hemionus). El objetivo de este estudio fue evaluar la tasa de depredación por puma (Puma concolor) en el borrego cimarrón y compararla con la del venado bura, para conocer la selección de presa del puma entre estas dos especies en un mismo hábitat, así como el impacto que podría tener en las poblaciones. Se plantea la hipótesis de que el puma como depredador oportunista no discrimina entre especies de ungulados simpátricos generando mayor impacto a la especie de menor densidad. Evaluamos la tasa de depredación por puma en 12 borregos cimarrones (10 hembras, 2 machos) y de 10 hembras adultas de venado bura con radio collares a través de micromorts y se comparó por medio una prueba de Wilcoxon, más la estimación de densidades de borrego y venados. Encontramos resultados similares en la causa específica de muerte por puma y supervivencia en ambas especies; $88 \%$ (8/9) muertes debido a depredación por puma en borrego cimarrón con una tasa de supervivencia mensual promedio de 0.79 y una tasa mensual de depredación que va del rango 0.17-0.30. En venado bura la depredación debido a puma fue $83 \%(5 / 6)$ con una tasa de supervivencia mensual promedio de 0.86 y una tasa de depredación por puma mensual en el rangos $0.10-0.25$. Sin embargo, al comparar con la tasa de depredación por puma encontramos una diferencia significativa $(Z=1.826 ; \mathrm{df}=6 ; \mathrm{P}=0.05)$ siendo el borrego cimarrón la presa más selecta. En este estudio se concluye que el puma seleccionó a la presa de menor densidad (borrego cimarrón) y por ende la más impactada en un hábitat simpátrico con venado bura.

Palabras claves: depredación por puma; borrego cimarrón; venado bura; Sierra Maderas del Carmen; tasa de supervivencia; translocación.

\section{REFERENCIAS}

Ackerman, B., Lindzey, F., \& Hemker, T. (1986). Cougar food habits in southern Utah. Journal of Wildlife Management, 48(1), 147-155.

Baker, R. (1956). Mammals of Coahuila Mexico. University of Kansas Publications: Kansas, E.U.A.: Museum of Natural History.

Bourbean-Lemieux, A., Festa-Bianchet, M., Gillard, J. M., \& Pelletier, F. (2011). Predator-driven component Allee effects in a wild ungulate. Ecology Letters, 14, 358-363.

Cashman, J. L., Peirce, M., \& Krausman, P. R. (1992). Diets of mountain lions in southwestern Arizona. The Southwestern Naturalist, 37(3), 324-326.
Chow, L. (1991). Population dynamics and movement patterns of bighorn sheep reintroduced to Sierra Nevada, California (Tesis de Maestría). California University, Davis, California.

Courchamp, F., Clutton-Brock, T., \& Grenfell, B. (1999). Inverse density dependence and the Allee effect. Tree, 14, 405-410.

Dickens, M. J., Delehanty, D. J., \& Romero, L. M. (2000). Stress: an inevitable component of animal translocation. Biological Conservation, 143, 1329-1341.

Elbroch, L. M., \& Wittmer, H. U. (2013). The effects of prey selection and specialization on less abundant prey in Patagonia. Journal of Mammalogy, 94(2), 259-268.

Ernest, H. B., Rubin, E. S., \& Boyce, W. M. (2002). Fecal DNA analysis and risk assessment of mountain lion predation of bighorn sheep. Journal of Wildlife Management, 66(1), 75-85.

Festa-Bianchet, M. (1991). The social system of bighorn sheep: grouping patterns, kinship and female dominance rank. Animal Behavior, 42, 71-82.

Haynes, C. L., Rubin, E. S., Jorgensen, M. C., Botta, R. A., \& Boyce, W. M. (2000). Mountain lion predation of bighorn sheep in the Peninsular Ranges, California. Journal of Wildlife Management, 64(4), 954-956.

Heisey, D. M., \& Fuller, T. K. (1985). Evaluation of survival and cause-specific mortality rates using telemetry data. Journal of Wildlife Management, 49(3), 668-674.

Hoban, P. A. (1990). A review of desert bighorn sheep in the San Andres Mountains, New Mexico. Desert Bighorn Council Transactions, 34, 14-22.

Holl, S. A., Bleich, V. C., \& Torres, S. G. (2004). Population dynamics of bighorn sheep in the San Gabriel Mountains, California, 1967-2002. Wildlife Society Bulletin, 32, 412-426.

Janke, T. S. (2015). Movements and survival of translocate desert bighorn sheep in the Bofecillos Mountains Transpecos, Texas (Tesis Maestría). Sul Ross University, Alpine, Texas.

Kamler, J. K., Lee, R. L., de Vos, J. C., Balard, W. B., \& Whitlaw, H. A. (2002). Survival and cougar predation of translocated bighorn sheep in Arizona. The Journal of Wildlife Management, 66(4), 1267-1227.

Krausman, P. R., \& Ables, E. D. (1981). Ecology of the Carmen mountain white-tailed deer. U. S. Department of Interior National Park Service; Washington, D. C. Scientific Monograph Series, 15, 65-73.

Lancia, R. A., Kendall, W. L., Pollock, K. H., \& Nichols, J. D. (2005). Estimating the number of animals in wildlife populations En C. E. Braun (Eds.), Techiques 
for wildlife investigations and management (pp. 117118). Bethesda, E.U.A.: The Wildlife Society.

Leopold, B. D., \& Krausman, P. R. (1986). Diets of 3 predators in Big Bend National Park, Texas. Journal of Wildlife Management, 50(2), 290-295.

Logan, K., \& Sweanor, L. (2001). Desert puma: evolutionary ecology and conservation of an enduring carnivore. Washington D. C., E.U.A.: Island press.

McKinney, B. P. (1996). Afield guide to Texas mountain lion. Austin, E.U.A.: Texas Park and Wildlife, Wildlife Division.

McKinney, B., \& Villalobos, J. (2005). Desert bighorn sheep reintroduction in Maderas del Carmen, Coahuila, México. Desert Bighorn Council Transactions, $48,46-49$.

McKinney, T., Smith, T., \& de Vos, J. C. (2006a). Evaluation of factors potentially influencing a desert bighorn sheep population. Wildlife Monographs, 164, 1-36.

McKinney, T., de Vos, J. C., Ballard, W. B., \& Boe, S. R. (2006b). Mountain lion predation of translocated desert bighorn sheep in Arizona. Wildlife Society Bulletin, 34(5), 1255-1263.

Mooring, M. S. (2004). Vigilance, predation risk, and the Allee effect in desert bighorn sheep. Journal of Wildlife Management, 68(3), 519-532.

Rominger, E. M. (2018). The Gordian knot of mountain lion predation and bighorn sheep. Journal of Wildlife Management, 82(1), 19-81.

Rominger, E. M, Whitlaw, H. A., Weybright, D. L., Dunn, W. C., \& Ballard, W. B. (2004). The influence of mountain lion predation on bighorn sheep translocation. The Journal of Wildlife Management, 68(4), 993-999.

Rosas-Rosas, O. C., Valdez, R., Bender, L. C., \& Daniel, D. (2003). Food habits of pumas in northwestern Sonora, Mexico. Wildlife Society Bulletin, 31, 528-535

Ross, P., Jalkotzy, M., \& Festa-Bianchet, M. (1997). Cougar predation on bighorn sheep in southwestern Alberta during winter. Canadian Journal Zoology, 74, 771-775.

Sánchez, O. (2005). Familia Bovidae, Borrego Cimarrón. En G. Ceballos \& O. Gissell (Eds.), Los Mamíferos silvestres de México (pp. 507-509). México D. F., México: Comisión Nacional para el Conocimiento y Uso de la Biodiversidad.

Schaefer, R. J., Torres, S. G., \& Bleich, V. C. (2000). Survivorship and cause-specific mortality in sympatric population of mountain sheep and mule deer. California Fish and Game, 86(2), 127-135.

Secretaría de Medio Ambiente y Recursos Naturales [SEMARNAT]. (2013). Programa de Manejo del Área de Protección de Flora y Fauna Maderas del Carmen, Coahuila, México. México D. F., México: Instituto Nacional de Ecología.

Secretaría de Medio Ambiente y Recursos Naturales [SEMARNAT]. (2010). Norma Oficial Mexicana NOM-059-SEMARNAT-2010 Diario Oficial de la Federación. Recuperado de http://www. profepa.gob. mx/innovaportal/file/435/1/NOM 059 SEMARNAT_2010.pdf

Simmons, N. M., \& Hansen, C. G. (1980). Population survey methods. En G. Monson \& L. Summer (Eds.), The Desert Sheep (pp. 270-271) Tucson, E.U.A.: University of Arizona Press.

Velázquez, R. (2012). Evaluación poblacional y del hábitat de un grupo de borregos cimarrones (Ovis canadensis mexicana Merriam, 1901), translocados en la Sierra Maderas del Carmen, Coahuila, México (Tesis de Maestría). U.A.N.L., Linares, México.

Villarreal, J. G. (1999). Venado Cola Blanca; manejo y aprovechamiento cinegético. Monterrey, México: Unión Ganadera Regional.

Villepique, J., Pierce, B., Bleich, V., \& Bowyer, R. (2011). Diet of cougar (Puma concolor) following a delaine in a population of mule deer (Odocoileus hemionus): lack of evidence for switching prey. The Southwestern Naturalist, 56(2), 187-192.

Wakeling, B. F., Lee, R., Brown, D., Thompson, R. Tluczek, M., \& Weisenberger, M. (2009). The restoration of desert big horn sheep in the Southwest, 1957-2007: factors influencing success. Desert Big Horn Council Transactions, 50, 1-17.

Wehausen, J. D. (1996). Effects of mountain lion predation on bighorn sheep in the Sierra Nevada and Granite Mountains of California, Wildlife Society Bulletin, 24(3), 471-479. 